\title{
Fibre Reinforced Polymer Composites for Structural Applications in Construction
}

\author{
Jun Deng, ${ }^{1}$ Marcus M. K. Lee, ${ }^{2}$ Baolin Wan, ${ }^{3}$ and Giuseppina Amato ${ }^{4}$ \\ ${ }^{1}$ Guangdong University of Technology, Guangzhou, China \\ ${ }^{2}$ Curtin University Sarawak, Miri, Malaysia \\ ${ }^{3}$ Marquette University, Milwaukee, WI, USA \\ ${ }^{4}$ Queen's University Belfast, Belfast, UK \\ Correspondence should be addressed to Jun Deng; jdeng@gdut.edu.cn
}

Received 28 May 2017; Accepted 28 May 2017; Published 2 July 2017

Copyright (C) 2017 Jun Deng et al. This is an open access article distributed under the Creative Commons Attribution License, which permits unrestricted use, distribution, and reproduction in any medium, provided the original work is properly cited.

In recent years, advanced fibre reinforced polymer (FRP) composites have become increasingly popular following the rapid growth in structural applications in construction around the world. These high-performance materials have been utilized in construction both for new structures and for strengthening/rehabilitation of existing buildings and bridges. Despite their widespread use, their full potential has still not been realized because of a number of fundamental concerns including high material costs, durability issues, bonding integrity, long-term interaction between loading and damage, and so forth. This special issue is aimed at providing a platform for the dissemination and discussion of recent research and achievements which address the issues on this topic from the theoretical and practical viewpoints.

A total of 13 original research papers were published in this special issue. These papers deal with materials and products, bond behavior, strengthening of concrete structures, and durability. Six papers focus on the mechanical and durability performances of FRP materials and products including BFRP bars, GFRP bars, steel-FRP bars, BFRP mesh, GFRP sheets, and basalt particle-filled SMC composites. Three papers report investigations on bond behavior of both FRP-concrete and FRP-steel after environmental exposure. Two more papers deal with prestressing systems designed to strengthen reinforced concrete beams and concrete-filled steel tubes and especially the prestress loss and bearing capacity. Theoretical and experimental studies on the durability behavior of FRP strengthened reinforced concrete beams subjected to fatigue loading and hygrothermal environment are reported in two papers as well.

These recent theoretical and experimental investigations that have been disseminated in this special issue address some critical fundamental issues, especially the long-term performance of both the FRP materials and strengthened structures. We expect that this special issue will be of interest to both researchers and engineers and contribute toward the developments in FRP applications in construction.

\section{Acknowledgments}

We greatly appreciate all the authors for their contributions and reviewers for their constructive comments and suggestions.

Jun Deng

Marcus M. K. Lee

Baolin Wan

Giuseppina Amato 

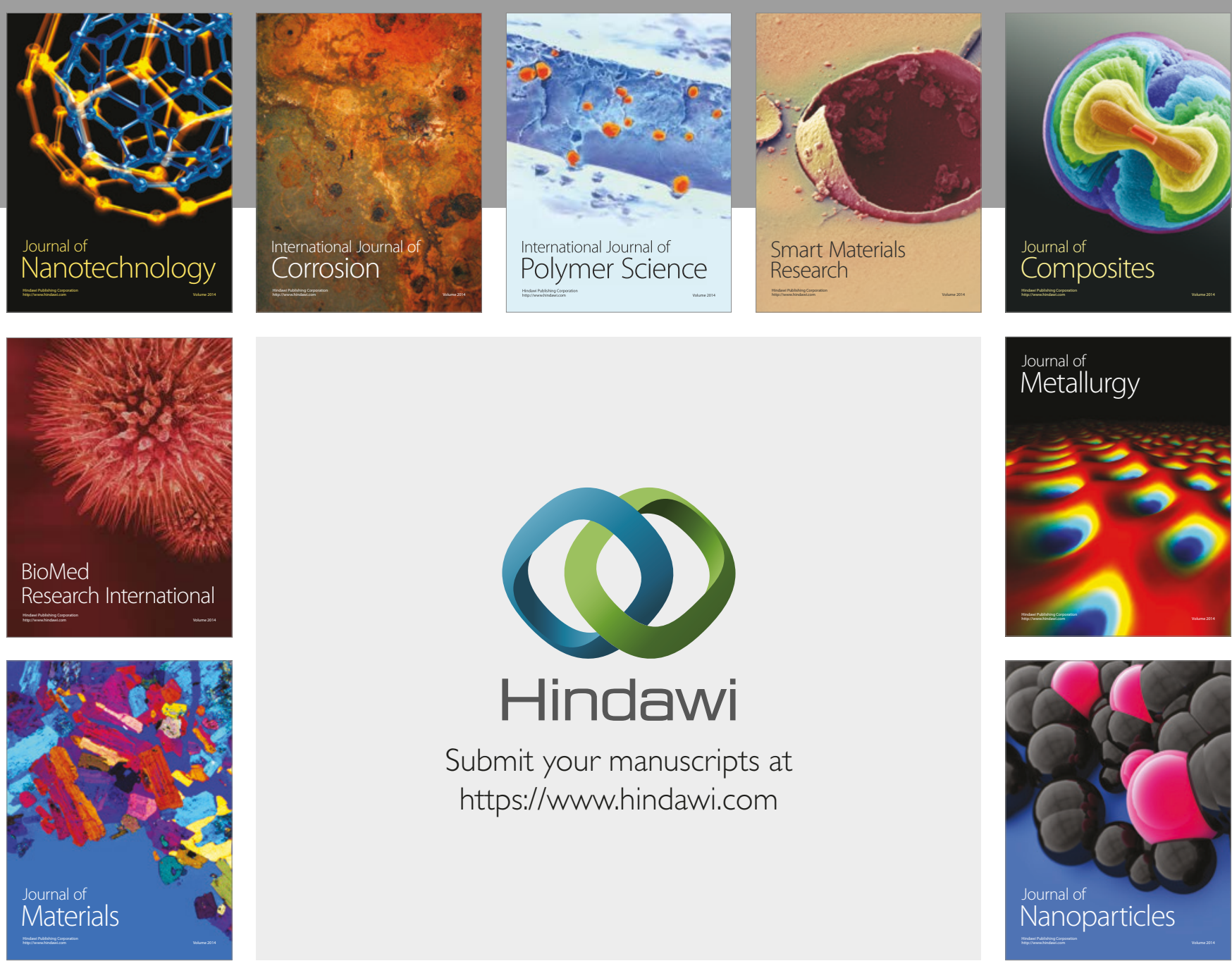

\section{Hindawi}

Submit your manuscripts at

https://www.hindawi.com
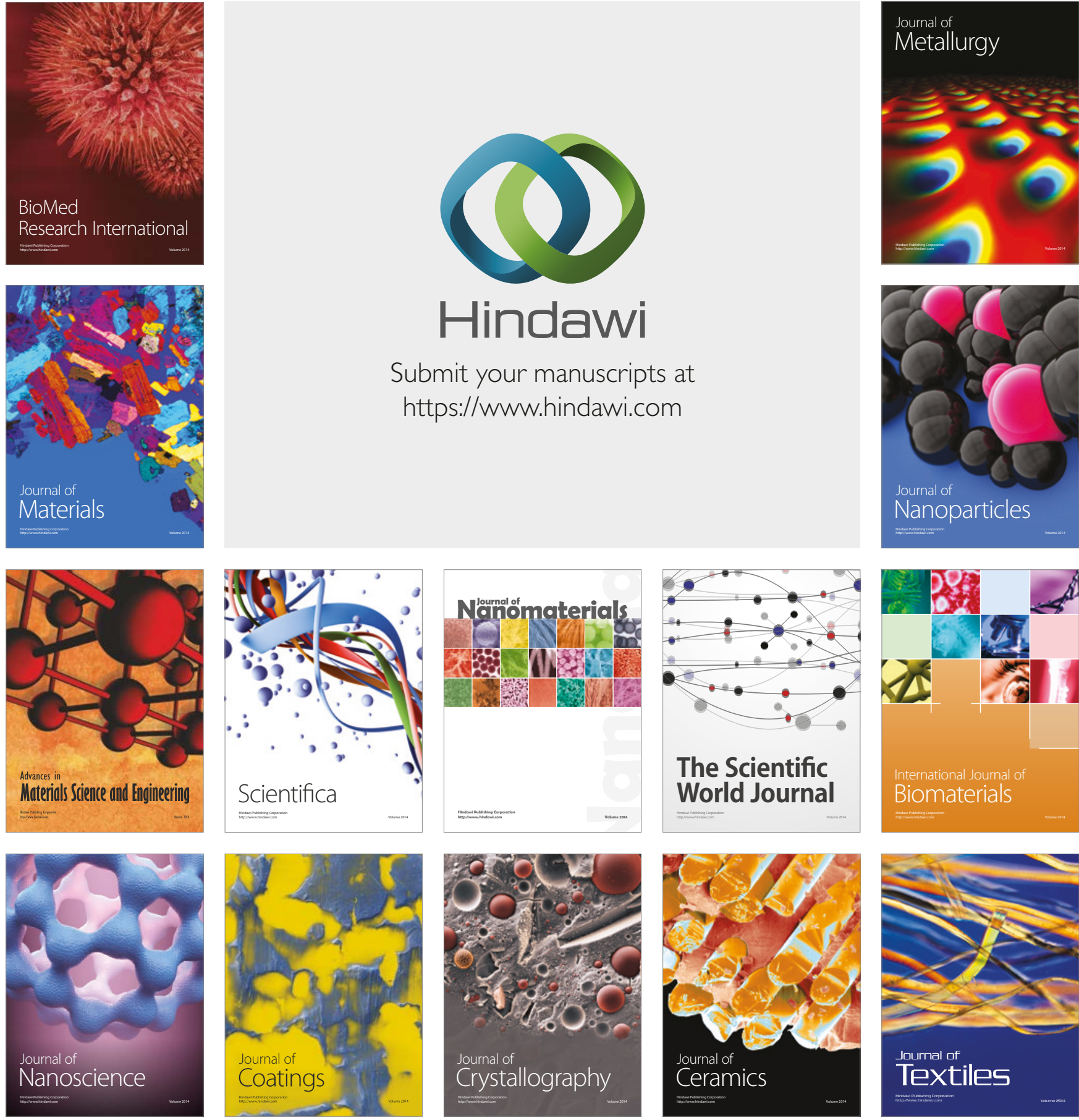

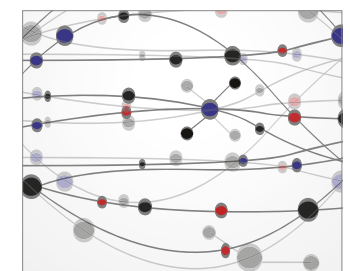

The Scientific World Journal
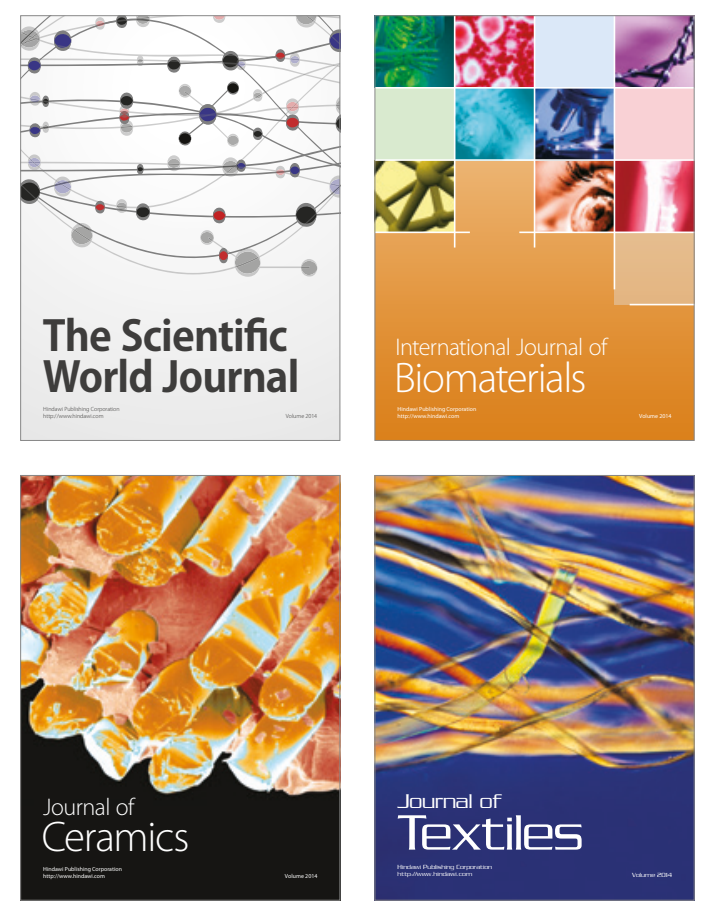